\title{
Characterization of hydroxyapatite laser ablation plumes by fast intensified CCD-imaging
}

\author{
P. Serra, J. Palau, M. Varela, J. Esteve, and J. L. Morenza \\ Departament de Física Aplicada i Electrònica, Universitat de Barcelona, Avda. Diagonal 647, E-08028 \\ Barcelona, Spain
}

(Received 1 August 1994; accepted 26 August 1994)

\begin{abstract}
ArF excimer laser pulses $(193 \mathrm{~nm}, 20 \mathrm{~ns}, 150 \mathrm{~mJ})$ have been focused on a hydroxyapatite (HA) target in similar conditions to those normally used for thin film deposition. Fast intensified CCD images of HA laser ablation plumes have been taken in vacuum and under different water vapor pressures ranging from 0.01 mbar to 1 mbar. Images of HA ablation in vacuum have shown a plume freely expanding at a constant velocity of 2.3 $\times 10^{6} \mathrm{~cm} / \mathrm{s}$. HA ablation under a water vapor pressure of $0.01 \mathrm{mbar}$ has revealed an expansion behavior very similar to that of ablation in vacuum. Images taken under a water vapor pressure of $0.1 \mathrm{mbar}$ have shown the formation of a shock structure in the plume. Finally, HA ablation under a water vapor pressure of 1 mbar has revealed the development of some irregularities in the shape of the plume.
\end{abstract}

\section{INTRODUCTION}

Hydroxyapatite thin films are specially adequate for bioactive coatings, mainly for Ti prostheses. Due to HA complex stoichiometry, pulsed laser deposition (PLD) has been revealed as a good technique for such a purpose. In order to obtain good crystalline films, the deposition process has been carried out under different reactive atmospheres. Diverse gas mixtures have been tried, such as $\mathrm{O}_{2}, \mathrm{O}_{2} / \mathrm{H}_{2} \mathrm{O}$, or $\mathrm{Ar} / \mathrm{H}_{2} \mathrm{O},{ }^{1-3}$ or merely using $\mathrm{H}_{2} \mathrm{O}$ alone. ${ }^{4}$ The results as obtained indicate that $\mathrm{H}_{2} \mathrm{O}$ is necessary in order to obtain thin films with good properties. Therefore, it seems interesting to analyze the HA ablation process under different water vapor atmosphere pressures, not only from a fundamental point of view but also to optimize the deposition conditions.

Similar studies have been carried out with $\mathrm{YBa}_{2} \mathrm{Cu}_{3} \mathrm{O}_{7-x}$ laser ablation under oxygen atmosphere at diverse pressures ${ }^{5-7}$ since oxygen pressure has been revealed as one of the most determinant technological parameters in high temperature superconductor thin film growth. These works show how the material ejected from the target by the laser pulse forms a plasma, also called "plume", whose expansion dynamics is analyzed accurately.

Among the different characterization techniques available, the one that shows more clearly this plume expansion is fast intensified CCD imaging. ${ }^{6,7}$ Due to its high spatial and temporal resolution, it allows one to visualize in detail the shape of the plume and its further evolution.

In this paper we present an analysis by fast intensified CCD imaging of the dynamics of the HA laser ablation process under vacuum and under diverse water vapor pressures in the range used in HA thin film deposition.

\section{EXPERIMENTAL}

A pellet made from HA powder pressed at $6 \times$ $10^{8} \mathrm{~Pa}$ pressure at room temperature was irradiated with an incident angle of $45^{\circ}$ by means of an $\mathrm{ArF}$ excimer laser beam (Lambda Physik LPX 205i, 193 nm, $20 \mathrm{~ns}$ ), limited by a $25 \mathrm{~mm}$ wide per $10 \mathrm{~mm}$ high mask, at an energy of $150 \mathrm{~mJ}$ per pulse, measured downstream from the mask. The pellet was placed at the focus of a $445 \mathrm{~mm}$ focal length spherical lens, in a high vacuum chamber evacuated by a turbomolecular pump to a base pressure of $5 \times 10^{-5}$ mbar. A water vapor atmosphere could be held in the ablation chamber thanks to a leak valve, in the range of pressures $0.01-0.1$ mbar.

Images were taken in a single shot mode with a gated CCD camera (ANIMATER-VI from ARP-France) $288 \times 384$ pixels, 8 bits dynamic range, intensified by means of a microchannel plate (MCP). The MCP aperture was delayed by a pulse generator triggered by a fast photodiode that was activated when the laser pulse impinged on it. This photodiode was placed between the laser cavity exit window and the beam limiting mask in order to be activated without deformation of the laser spot on the target. The gain on the MCP was variable and set just short of saturation, to take advantage of the full range of each pixel on the CCD array.

Under vacuum conditions the plume was viewed parallel to the target in two different directions, perpendicular to both axes of the laser spot, in order to describe a three-dimensional expansion from two-dimensional images. All other series of images, at diverse background 
water vapor pressures, were taken only in one direction, perpendicularly to the largest axis of the laser spot. In all these cases the imaged region extended $6 \times 4.5 \mathrm{~cm}^{2}$.

\section{RESULTS AND DISCUSSION}

The series of images corresponding to ablation of HA under vacuum conditions $\left(5 \times 10^{-5} \mathrm{mbar}\right)$ viewed perpendicularly to the largest axis of the laser spot [Fig. 1(a)] show the existence of two well-defined plasma clouds separated by a thin dark region. Both clouds expand freely without intersection and without changes in their shapes, i.e., developing a self-similar expansion. The smallest cloud always remains between the largest one and the target, and its appearance is that of an expanding layer. It shows weaker brightness than the other one, with an almost inappreciable lateral expansion. On the other hand, the largest cloud, very bright, exhibits a plumelike shape, with a luminous a)

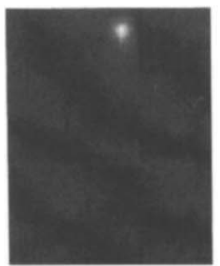

$400 \mathrm{~ns}$

b)

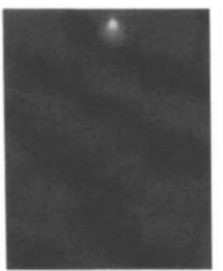

$400 \mathrm{~ns}$

c)

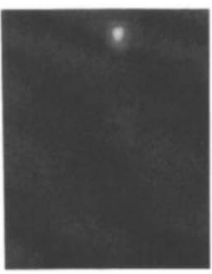

$400 \mathrm{~ns}$

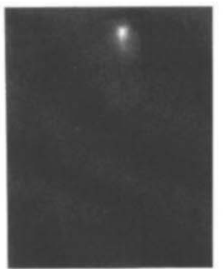

$400 \mathrm{~ns}$

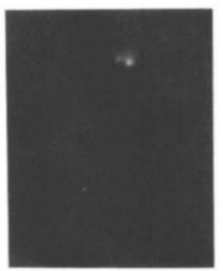

$1 \mu \mathrm{s}$

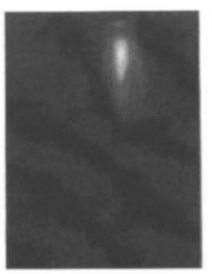

$1 \mu \mathrm{s}$

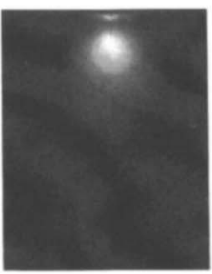

$1 \mu \mathrm{s}$

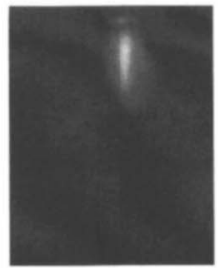

$1 \mu \mathrm{s}$

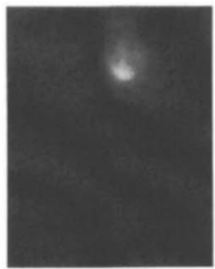

$1 \mu \mathrm{s}$

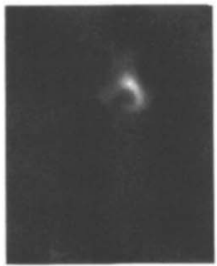

$6 \mu \mathrm{s}$

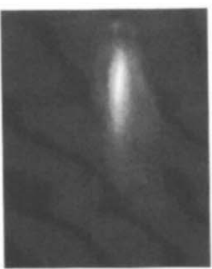

$1.6 \mu \mathrm{s}$

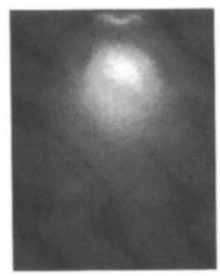

$1.6 \mu \mathrm{s}$

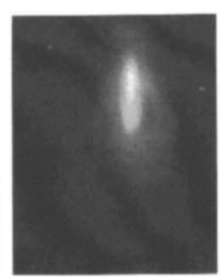

$1.6 \mu \mathrm{s}$

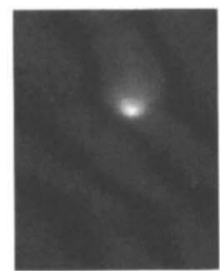

$2 \mu s$

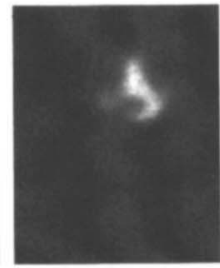

$10 \mu \mathrm{s}$

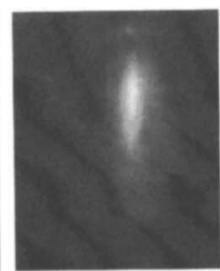

$2 \mu \mathrm{s}$

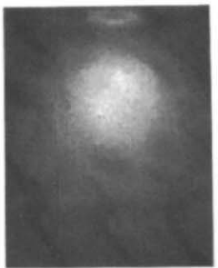

$2 \mu \mathrm{s}$

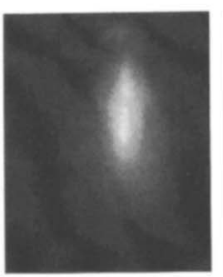

$2 \mu \mathrm{s}$

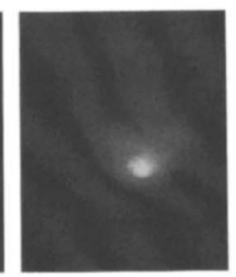

$6 \mu \mathrm{s}$

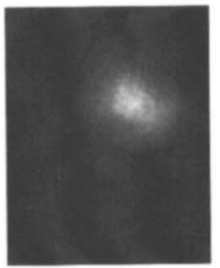

$22 \mu \mathrm{s}$

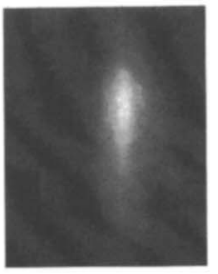

$2.5 \mu \mathrm{s}$

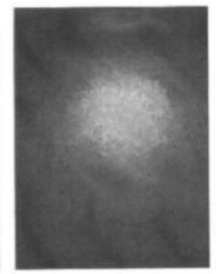

$2.5 \mu \mathrm{s}$

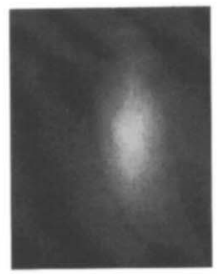

$2.5 \mu \mathrm{s}$

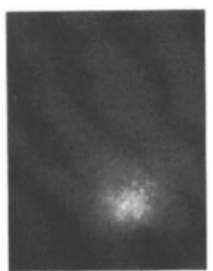

$18 \mu \mathrm{s}$

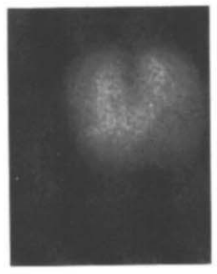

$100 \mu s$

FIG. 1. Time evolution of HA laser ablation plumes under vacuum (a) viewed perpendicularly to the major axis of the laser spot, (b) viewed perpendicularly to the minor axis of the laser spot, and under water vapor (viewed perpendicularly to the major axis of the laser spot) at a pressure of (c) 0.01 mbar, (d) 0.1 mbar and (e) 1 mbar. (The MCP gain is variable and increases with delay time for each series of images.) 
maximum in its inner part and an expansion front whose brightness is smoothly attenuated. Two symmetrically disposed lateral lobes are visible, expanding with the plume, probably originated by the inhomogeneity of the laser spot along its largest axis.

The same series viewed perpendicularly to the minor axis of the laser spot [Fig. 1(b)] also reveals the presence of both plasma clouds with the same characteristics as the former case, but with a very pronounced lateral expansion of the largest one. Its shape is not plumelike, but almost circular and no lobes are visible. Further observations of the laser spot on the HA target have shown no inhomogeneities in its smallest axis.

This greater lateral expansion in the direction of the smallest axis of the spot in laser ablation generated plasmas has been described by several theoretical models ${ }^{8-10}$ that proceed by solving the hydrodynamical equations for an ellipsoidal monoatomic gas cloud that freely expands in vacuum.

Plume luminous intensity profiles are displayed in Fig. 2 for the case of a plume viewed perpendicularly to the largest axis of the spot, in order to describe the dynamics of the laser-generated plasma. Two intensity maxima can be observed there, a small one, close to the target, corresponding to the light emission of the smaller plasma cloud, and a large one, whose intensity decreases asymptotically to zero, corresponding to the plumelike cloud. It has always been taken as the plume expansion front that point where light intensity reaches $10 \%$ of the largest intensity maximum. Plots of this front position versus time have been depicted in Figs. 3(a)

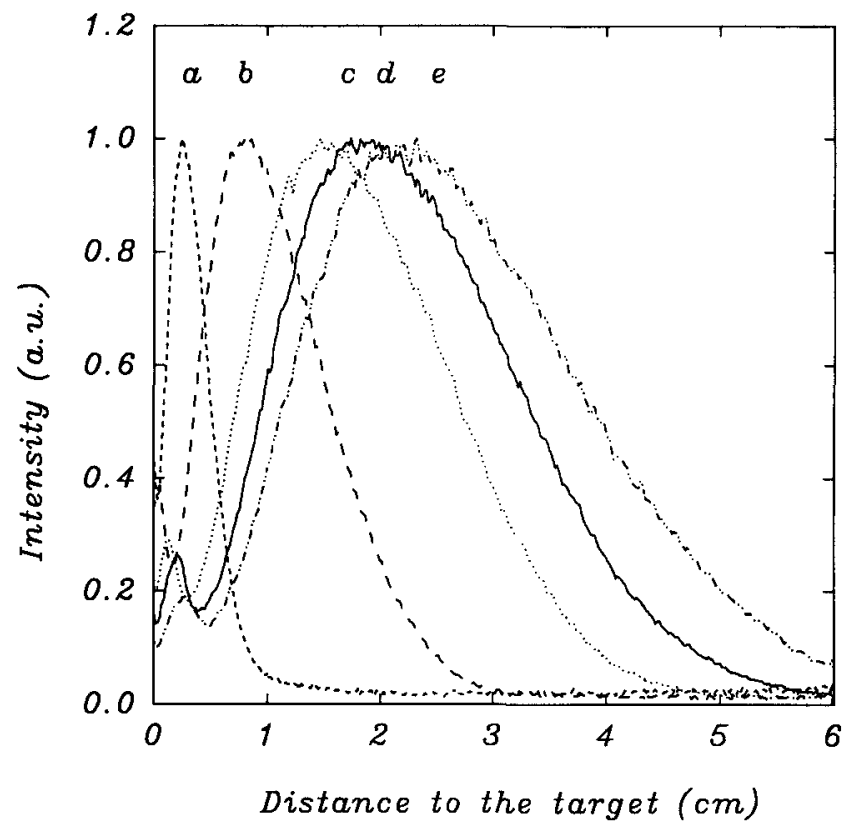

FIG. 2. Normalized intensity profiles of the plume in vacuum HA ablation at different instants of time after the laser pulse: (a) $400 \mathrm{~ns}$, (b) $1 \mu \mathrm{s}$, (c) $1.6 \mu \mathrm{s}$, (d) $2 \mu \mathrm{s}$, and (e) $2.5 \mu \mathrm{s}$.

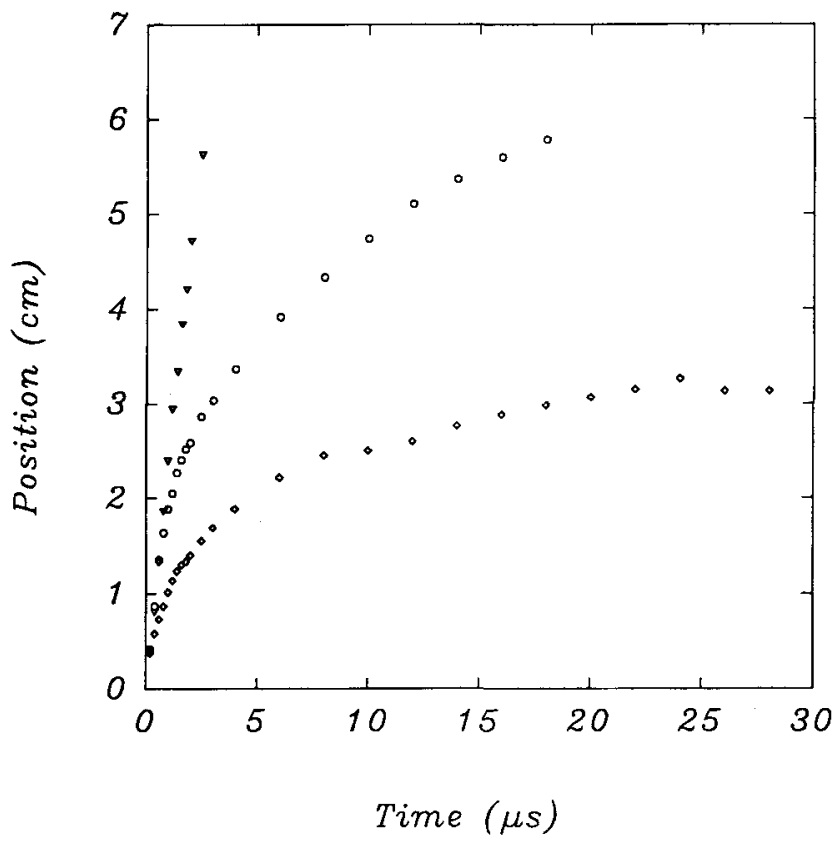

FIG. 3. Position of the plume front measured from the target versus time for HA ablation under (a) vacuum $(\nabla)$ and water vapor at (b) 0.1 mbar $(\bigcirc)$ and (c) 1 mbar $(\diamond)$.

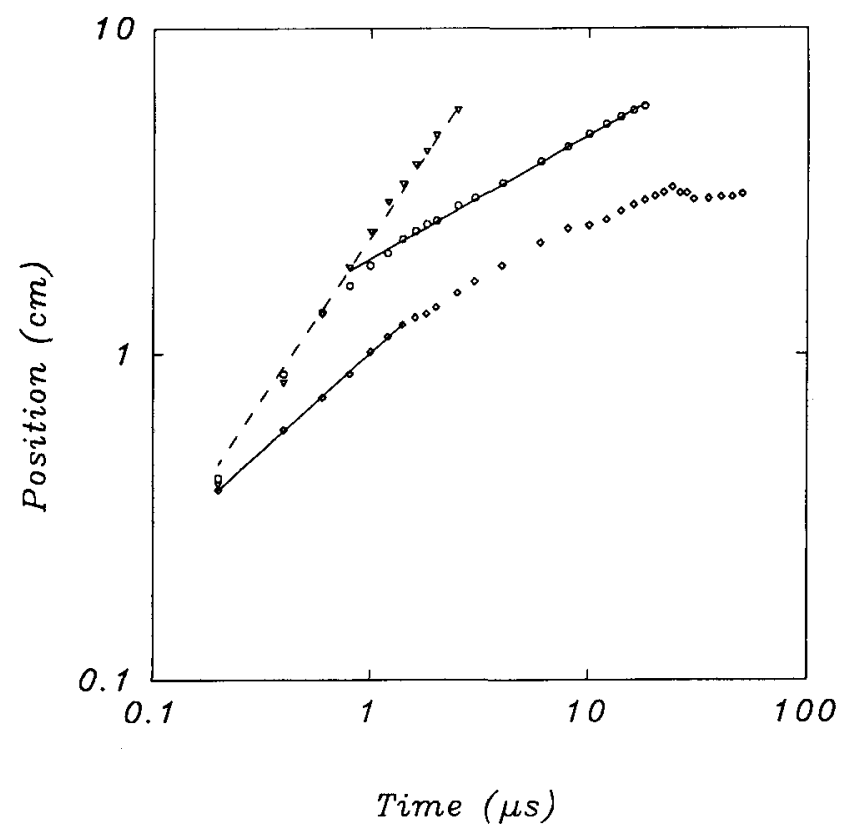

FIG. 4. Logarithmic plots of the position of the plume front measured from the target versus time for HA ablation under (a) vacuum $(\nabla)$ and water vapor at (b) $0.1 \mathrm{mbar}(\mathrm{O})$ and (c) 1 mbar $(\diamond)$.

and 4(a). As they can be well fitted by a straight line, it can be concluded that the motion of the leading edge of the plume is at constant velocity. The speed for this expansion front has been found to be $2.3 \times 10^{6} \mathrm{~cm} / \mathrm{s}$, the order of magnitude of which is in agreement with those 
found by other authors for ablation of several materials under vacuum in similar conditions. ${ }^{6,7,11}$

The existence of the small plasma cloud near the target has been observed with fast intensified CCD imaging by Geohegan ${ }^{6}$ for $\mathrm{YBa}_{2} \mathrm{Cu}_{3} \mathrm{O}_{7-x}$ laser-generated plumes. He has described it as an essentially stationary component that occupies a region out to $1 \mathrm{~mm}$ from the target surface. However, the plots of the position of the smaller maximum versus time [Fig. 5(a)] reveal that, for HA ablation plumes, this component is not stationary, but it expands into vacuum at a constant velocity of $1.5 \times 10^{5} \mathrm{~cm} / \mathrm{s}$. Eryu et al. ${ }^{12}$ have found by transient optical transmittance measurements different populations in plumes generated by ablation of $\mathrm{YBa}_{2} \mathrm{Cu}_{3} \mathrm{O}_{7-x}$ that expand at different velocities of the order of magnitude of the values presented here. It is possible to explain the presence of two clouds instead of a single plume by means of gas-phase segregation effects. These effects make a plasma, initially composed of two different mass species, become, as it expands, two separated plasma clouds, each composed of one single species. Both clouds, when completely segregated, expand freely with constant velocity, which is larger for the cloud of the lighter mass species. Urbassek and Sibold ${ }^{13}$ have developed a Monte Carlo simulation code that describes this process for pulsed desorption experiments where a two species plume is generated.

The series of images corresponding to ablation under water vapor pressures have been taken only in a view perpendicular to the major axis of the laser spot. In particular, those taken at 0.01 mbar [Fig. 1(c)] show an expansion dynamics very similar to the case of HA

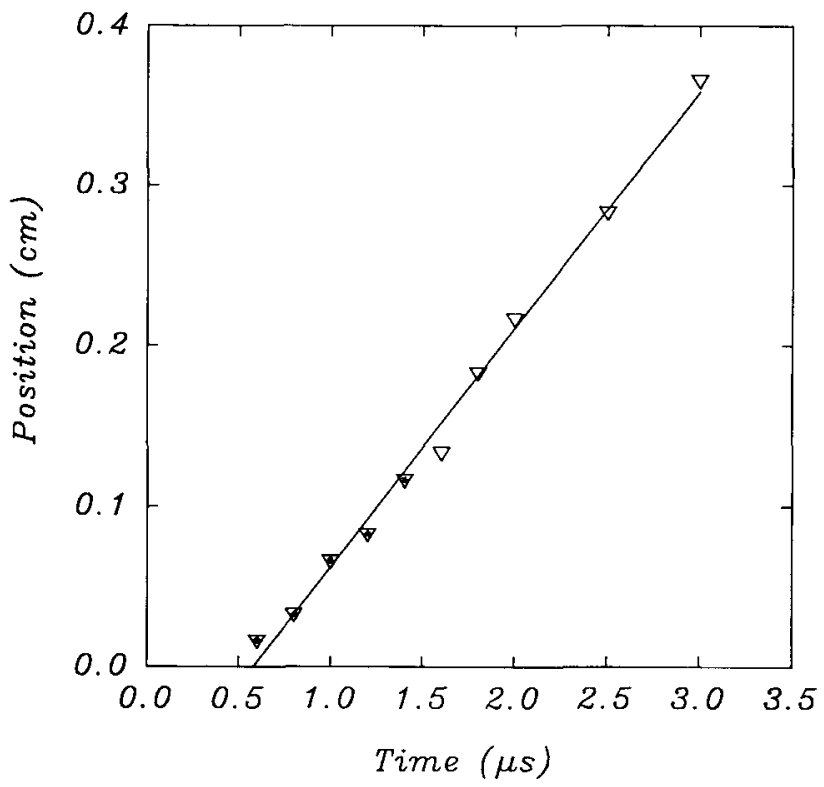

FIG. 5. Position of the small intensity maximum measured from the target versus time for HA ablation under (a) vacuum $(\nabla)$ and water vapor at (b) 0.1 mbar (O). ablation under vacuum. The shape of the plumes at this pressure is almost the same, although lateral expansion is greater and no lobes are visible. Furthermore, the effect of collisions with the background gas slightly confines the species in the front, developing a sphericallike shape, not observed in the case of vacuum, without formation of a shock structure. This confinement has no detectable effects on the motion of the plume, at least for the distances from the target studied here, since the velocity of the leading edge has been found to be the same as that observed under vacuum conditions and also on the smaller plasma cloud that is also present in this case.

The series of images taken at a water vapor pressure of 0.1 mbar [Fig. 1(d)] shows an expansion behavior radically different from that found in the cases mentioned above. At the beginning of the expansion, the first $800 \mathrm{~ns}$ after the laser pulse, it can be seen how the background gas confines the species of the plasma just at the leading edge of the plume, resulting in a very bright spherical front of expansion. After this period of time, no appreciable changes can be observed in the shape of the plume except an increase in relative brightness in the initially dark region between the plume and the target. Furthermore, it also appears in the small plasma cloud near the target under these pressure conditions, although its brightness is weaker than in the experiments described above and also is its lifetime.

The luminous intensity profiles displayed in Fig. 6 show very clearly the formation of the bright lead-

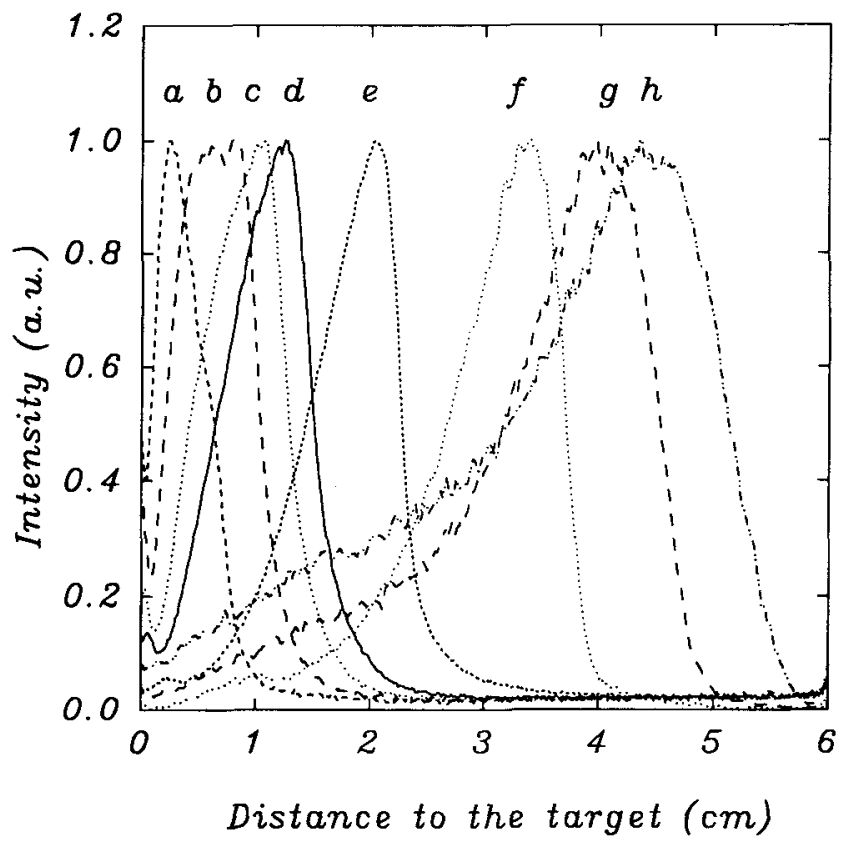

FIG. 6. Normalized intensity profiles of the plume in HA ablation under a water vapor pressure of $0.1 \mathrm{mbar}$ at different instants of time after the laser pulse: (a) $400 \mathrm{~ns}$, (b) $600 \mathrm{~ns}$, (c) $800 \mathrm{~ns}$, (d) $1 \mu \mathrm{s}$, (e) $2 \mu \mathrm{s}$, (f) $6 \mu \mathrm{s}$, (g) $12 \mu \mathrm{s}$, and (h) $18 \mu \mathrm{s}$. 
ing edge in the plasma. The intensity maximum in the plume moves away its inner part, near the target, toward the front, until it develops a sharp transition from its maximum value to zero intensity positions. This transition in the profiles reveals the formation of a shock structure. Most species in the plasma have been confined by the background gas near the leading edge, resulting in a bright front whose light emission is due to collisions that take place among the ablated species and between these and those of the reactive atmosphere. This shock structure can be maintained while the pressure in the plume front is greater than the pressure of the background gas.

Plots of the distance from the target to the leading edge of the plume versus time [Fig. 3(b)] show that expansion under a water vapor pressure of $0.1 \mathrm{mbar}$ is not at constant velocity, although for instants of time prior to $1 \mu \mathrm{s}$ the position of the front coincides with that for the case of HA ablation in vacuum. After this time the plume is decelerated by the background atmosphere, resulting in a dynamical behavior radically different from in vacuum conditions. The expansion of the shock structure can be described by hydrodynamical equations whose solution, for a spherical shock wave, is ${ }^{14}$ :

$$
R=\xi_{0}\left(E / \rho_{0}\right)^{1 / 5} t^{2 / 5},
$$

where $R$ is the distance between the target and the plume front, $\xi_{0}$ is a constant, $E$ is the energy of the ablated particles, $\rho_{0}$ is the undisturbed background gas density, and $t$ is the instant of time considered after the laser impingement. Then, the expression

$$
R=A t^{\alpha}
$$

has been used to fit the experimental measurements [Fig. 4(b)] for $t>800 \mathrm{~ns}$, when the shock structure has been completely developed. It has been found $\alpha=0.38$, a value close to $\alpha=0.4$, the one predicted by the theory. Similar results have been obtained for $\mathrm{YBa}_{2} \mathrm{Cu}_{3} \mathrm{O}_{7-x}$ laser ablation under an oxygen background pressure. ${ }^{6,7,15}$ The shock wave model, just described above, does not apply for those instants of time prior to the formation of the shock structure $(t \leqslant 800 \mathrm{~ns})$, during which the plume expands at a constant velocity of $2.3 \times 10^{6} \mathrm{~cm} / \mathrm{s}$. Although this speed has the same value as that found under vacuum conditions, the behavior of the plume is not that of a free expansion as it can be seen in the profiles displayed in Figs. 6(a)-6(c). These correspond to the formation of the shock structure and reveal how the background gas confines the ejected particles in the leading edge until this shock structure is completely formed.

The expansion of the small plasma cloud near the target can also be observed from inspection of the profiles displayed in Fig. 6. In this case it is also possible to appreciate the existence of this small maximum although its brightness is lower, in magnitude and with respect to the principal maximum, than under vacuum conditions. In order to analyze the dynamics of its expansion, plots of its position versus time have been depicted in Fig. 5(b). Surprisingly, no changes are appreciable with respect to the motion of this small cloud when ablation is carried out in vacuum, as if it were screened by the plume.

Images taken at a water vapor pressure of $1 \mathrm{mbar}$ (Fig. 1(e)] show the whole material released from the target confined in an irregular asymmetric leading edge after the first $200 \mathrm{~ns}$ after the laser pulse. A shock structure has been developed, very different from the spherical shock wave found at 0.1 mbar. Such irregular shapes are not due to turbulences since images are completely reproducible. These irregularities completely disappear after $22 \mu \mathrm{s}$, as the plume stops, developing a spherical front whose luminous intensity distribution evolves in time. The emitting plasma is still visible $200 \mu \mathrm{s}$ after the laser impingement with no changes in either its shape or position during its following evolution, diminishing its brightness, until it completely disappears $1 \mathrm{~ms}$ later. Under such a high water vapor pressure, the small plasma cloud near the target has not been observed. Other irregular plume shapes have been reported on the ablation of graphite in a high pressure (400 mbar) atmosphere of Xe. ${ }^{16}$

Although there is not a clear definition of the plume leading edge for such a non-self-similar motion, plots of its position versus time have been depicted in Figs. 3(c) and 4(c), using the criteria described above. Three different regions can be pointed out by simple inspection of Fig. 4(c). The first, ranging from $200 \mathrm{~ns}$ to $1.4 \mu \mathrm{s}$, shows a set of well-aligned points that can be described by Eq. (2) with $\alpha=0.6$, an intermediate value between $\alpha=0.4$, corresponding to a spherical shock wave, with $\alpha=1$, for the case of expansion in vacuum. In the second one, ranging from $1.6 \mu \mathrm{s}$ to $26 \mu \mathrm{s}$, although the results are not well correlated and a good fit is not possible, the behavior seems to be similar to that found for HA ablation under a water vapor pressure of 0.1 mbar. Finally, in the third one, after $26 \mu \mathrm{s}$, the plume is almost stopped, beginning what seems to be a diffusion regime.

\section{CONCLUSION}

Fast intensified-CCD images have revealed that HA laser ablation plumes expand freely, at constant velocity, up to water vapor pressures of the order of 0.01 mbar. These plumes are followed by a second little plasma cloud that expands at a velocity one order of magnitude lower than the former, suggesting the existence of gasphase segregation effects. The different lateral expansion 
of the plume in the directions of both laser spot axes has also been shown.

At a water vapor pressure of $0.1 \mathrm{mbar}$, the expansion of the plume can be described, when a shock structure has been developed, by a spherical shock wave model. The motion of the second little plasma cloud seems to be screened by the plume.

Finally, at a water vapor pressure of 1 mbar, the plumes show irregular asymmetrical shapes, not due to turbulences since they are completely reproducible. Although it is not possible to give a precise definition of the plume front in this case, we have attempted to describe its dynamical behavior. During the first $1.4 \mu \mathrm{s}$, the plume shows an expansion regime completely different from those found for the other pressures under study. After this time and until $26 \mu \mathrm{s}$ after the laser pulse, its dynamics is quite similar to that of ablation under a water vapor pressure of $0.1 \mathrm{mbar}$, to finally get into what seems a diffusion regime. No second little plasma cloud has been observed in this case.

\section{ACKNOWLEDGMENTS}

This work has been supported by CICYT of the Spanish Government (project MAT94-0264) and European Economic Community (contract VALUE CCM304). The authors acknowledge the technical assistance of G. Blanchot and the Unitat Tècnico-mecànica of the Universitat de Barcelona.

\section{REFERENCES}

1. P. Baeri, L. Torrisi, N. Marino, and G. Foti, Appl. Surf. Sci. 54, 210 (1992).

2. C. M. Cotell and K.S. Grabowski, MRS Bull. 17 (2), 44 (1992).
3. G. Sardin, F. Sánchez, M. Varela, and J. L. Morenza, Third European East-West Conference of the E-MRS, Symposium on Biomaterials, Strasbourg, France (1992).

4. G. Sardin, M. Varela, and J. L. Morenza, in Hydroxyapatite and Related Materials, edited by P.W. Brown and B. Constantz (CRC Press, Boca Raton, FL, 1994), p. 225.

5. K. Scott, J. M. Huntley, W. A. Philips, J. Clarke, and J. E. Field, Appl. Phys. Lett. 57, 922 (1990).

6. D. B. Geohegan, Appl. Phys. Lett. 60, 2732 (1992).

7. J. Palau, P. Serra, M. Varela, J. Esteve, F. Sánchez, and J.L. Morenza, in Laser Ablation: Mechanisms and Applications II, edited by J.C. Miller and D. B. Geohegan (American Institute of Physics, New York, 1994), p. 427.

8. J. Dawson, P. Kaw, and B. Green, Phys. Fluids 12, 875 (1969).

9. R. K. Singh, O.W. Holland, and J. Narayan, J. Appl. Phys. 68, 233 (1990).

10. J.C.S. Kools, T.S. Baller, S.T. De Zwart, and J. Dieleman, J. Appl. Phys. 71, 4547 (1992).

11. R. M. Gilgenbach and P. L. G. Ventzek, Appl. Phys. Lett. 58, 1597 (1991).

12. O. Eryu, K. Murakami, K. Masuda, K. Shihoyama, and T. Mochizuki, Jpn. J. Appl. Phys. 31, 286 (1992).

13. H. M. Urbassek and D. Sibold, Phys. Rev. Lett. 70, 1886 (1993).

14. Y. B. Zel'dovich and P. Raizer, Physics of Shock Waves and High Temperature Hydrodynamic Phenomena (Academic, New York, 1966), Vol. 1, p. 94.

15. P. E. Dyer, A. Issa, and P. H. Key, Appl. Surf. Sci, 46, 89 (1990).

16. A. A. Puretzky, D. B. Geohegan, R. E. Haufler, R. L. Hettich, X. Y. Zheng, and R. N. Compton, in Laser Ablation: Mechanisms and Applications $I$, edited by J.C. Miller and D. B. Geohegan (American Institute of Physics, New York, 1994), p. 365. 